\title{
Kawasaki syndrome
}

\section{T A Fernando ${ }^{1}$}

Sri Lanka Journal of Child Health, 2000; 29: 29-30

(Key words: Kawasaki syndrome)

Dr. Tomisaku Kawasaki of Tokyo, Japan first described Kawasaki Syndrome (KS) in $1967^{1}$. The basic pathology of the disease is that of a multisysterm vascultis and almost exclusively it affects young children ${ }^{2}$. Apart from fever, within the first five days the child may have conjunctival injection, mouth changes, an erythematous rash, changes in the hands and feet and unilateral cervical lymphadenopathy. In order to diagnose $\mathrm{KS}$ the patient should exhibit 4 of the 5 features mentioned in addition to fever ${ }^{3}$. By far the most important and dangerous complication is the development of coronary artery aneurysms ${ }^{4}$. Three cases of Kawasaki syndrome (KS) are described to show the importance of a high "index of suspicion" of KS when dealing with a young child with fever.

\section{Case one}

A three and half year old boy presented with cough and fever of 2 days duration. He was prescribed erythromycin and ventolin expectorant. On the same day he developed severe abdominal pain and was admitted to a private nursing home. Since the abdominal examination was negative it was assumed that the abdominal pain was due to erythromycin and it was discontinued and he was placed on amoxycillin.

On admission, the total white cell count was $11.7 \mathrm{x}$ $10^{9} / \mathrm{L}$ (N 73, L 19, E 06, M 02\%). Haemoglobin was $11.4 \mathrm{~g} / \mathrm{dl}$. The platelet count was $337 \times 10^{9} / \mathrm{L}$. On the third day he developed loose stools and the abdominal pain continued. The stools full report, culture and rotavirus test were all negative. At this time he was quite irritable and complained of severe neck pain, left hip pain and abdominal distension. Due to the hip pain he was unable to stand up. A surgical consultation was made to rule out an acute abdomen. X-rays of abdomen, chest, neck and hips were normal. Ultrasound scans of abdomen and hips were also normal.

Around the fourth day he developed a macular erythematous rash on his trunk that lasted 2 days. His

\footnotetext{
${ }^{1}$ Consultant Paediatrician
}

lips were cherry red, cracking and bleeding. He also had bilateral non-purulent conjunctivitis. As the temperature was still quite high on the 5th day, a partial FUO workup was done, the amoxycillin was discontinued and he was placed on intravenous ceftriaxone. Blood culture, urine culture and stool culture were negative. The urine full report and SAT were negative. The total white cell count was $14.6 \mathrm{x}$ $10^{9} / \mathrm{L}$ ( $\mathrm{N} 66, \mathrm{~L} 30, \mathrm{E}$ 04\%). Haemoglobin was $9.3 \mathrm{~g} / \mathrm{dI}$. The haematocrit was $28 \%$. The platelet count was $488 \times 10^{9} / \mathrm{L}$. The ESR was $80 \mathrm{~mm}$. The blood picture was normal. The SGPT was $50 \mathrm{IU} / \mathrm{L}$.

On the 8th day of hospitalization the child still had a temperature of $101^{0} \mathrm{~F}$, red cracking lips, slight cough, left hip pain and irritability. Rheumatoid factor, anti nuclear antibody and LE cells were negative. The ESR was $90 \mathrm{~mm}$. The white cell count was $16 \mathrm{x}$ $10^{9} / \mathrm{L}$ (N 68, L 30, E 02\%). Haemoglobin was 9.lg/dI. The haematocrit was $27.7 \%$. The platelet count was $701 \times 10^{9} / \mathrm{L}$. Peeling of palms and soles were noticed about this time. At this stage a diagnosis of KS was made.

An echocardiogram (ECHO) was done which was fortunately normal. The child was placed on aspirin $100 \mathrm{mg} / \mathrm{kg}$. Since the diagnosis was made on the 11th or 12th day, no intravenous immunoglobulin (IVIG) was given. His temperature settled dramatically in 24 hours and he was sent home for outpatient follow up. Aspirin was continued for 6 weeks and discontinued when the ESR and platelet counts were normal. A repeat $\mathrm{ECHO}$ after 4 weeks was negative.

\section{Case two}

An eight year old boy developed fever 3-4 days following an appendicectomy. He also had a purulent discharge from his incision. Assuming that the fever was due to a secondary infection, he was given intravenous cefotaxime and metronidazole. The temperature subsided for one day and then shot up to $103^{\circ} \mathrm{F}$ and he developed a measles like rash on face and trunk. I was consulted at this stage. He appeared agitated, febrile but cooperative. There was a macular erythematous confluent rash on face and chest, red cracked bleeding lips, bilateral non-purulent 
conjunctivitis and somewhat swollen hands without induration. He had swollen but soft abdomen with a slightly purulent discharge from the appendicectomy incision. The culture of discharge revealed no growth. The white cell count was $7.6 \times 10^{9} / \mathrm{L}$ (N 76, L 20, E 01, M 04\%). Haemoglobin was 10g/dI. The haematocrit was $27 \%$. The platelet count was $466 \mathrm{x}$ $10^{9} / \mathrm{L}$. SGPT was $158 \mathrm{IU} / \mathrm{L}$. The urine full report was normal. The ESR was $10 \mathrm{~mm}$.

A diagnosis of $\mathrm{KS}$ was made and the child was started on aspirin $100 \mathrm{mg} / \mathrm{kg}$ and infusion of intravenous immunoglobulin at $2 \mathrm{~g} / \mathrm{kg}$ was started within 24 hours. It was given over a period of 12 hours. An ECHO done next day was normal. Antibiotics were stopped on the same day. The temperature subsided in 24 hours. He was sent home for outpatient follow up. His appetite and general condition improved remarkably. A repeat ECHO after 3 weeks was normal. His aspirin therapy was stopped after 5 weeks when the platelet counts normalised.

\section{Case three}

A two years and three months old boy was seen by me on the 12th day of fever after having been discharged from a private hospital with a diagnosis of "viral fever with urticaria". He had developed fever of 3 days duration followed by an erythematous macular rash. He was admitted to hospital to rule out dengue fever. He was discharged from hospital after 5 days. Since the fever persisted he was brought to me. He appeared ill, lethargic with a temperature of $100^{\circ} \mathrm{F}$. He had a fading, slightly macular erythematous rash on face, red cracking lips, strawberry tongue, conjunctival injection and slightly swollen (non-pitting) peeling palms and soles. The white cell count was $11.8 \times 10^{9} / \mathrm{L}$ (N 74, L 25, E $01 \%$ ). Haemoglobin was $11.3 \mathrm{~g} / \mathrm{dI}$. The haematocrit was $36.6 \%$. The platelet count was $502 \times 10^{9} / \mathrm{L}$. The SGPT was $195 \mathrm{IU} / \mathrm{L}$. ESR was $22 \mathrm{~mm}$.

Based on the clinical features and lab tests a diagnosis of KS was made. An ECHO done on the day of consultation was negative. He was treated with aspirin $100 \mathrm{mg} / \mathrm{kg}$ and treatment was stopped roughly after 4 weeks. Intravenous immunoglobulin was not given. A follow up ECHO was negative.

\section{Discussion}

Being a self-limiting disease $\mathrm{KS}$ can easily be missed. Because of the possible coronary complications it is important to make the diagnosis early and implement proper therapy. Even through only $20 \%$ will develop coronary complications, by diagnosing the disease and giving aspirin/IVIG the patient improves clinically in $24-28$ hours $90 \%$ of the time.

Diagnosis is based in clinical features taken together with laboratory findings and by excluding other conditions that may have similar presentation.

In conclusion, one must always consider $\mathrm{KS}$ in all young children who have fever of uncertain origin as there is effective treatment now.

\section{References}

1. Kawasaki T, Kosaki F, Okawas et al. A new infantile acute febrile mucocutaneous lymph node syndrome prevailing in Japan. Paediatrics, 1974; 54:271-6.

2. Fujiwara H, Hamashima Y. Pathology of the heart in Kawasaki disease. Paediatrics, 1978; 61:100-7.

3. American Heart Association Committee on Rheumatic fever and Kawasaki Disease; Diagnostic guidelines for Kawasaki Disease. $\mathrm{Am}$ J Dis Child, 1988; 144:1218.

4. Kato H, Inoue 0, Akagi $\mathrm{T}$ Kawasaki disease: cardiac problems and management. Paed Rev, 1988; 9:209-17. 
